\title{
Laser Speckle Analysis Synchronised with Cardiac Cycle
}

\author{
Pavel Zakharov ${ }^{1,2^{*}}$, Frank Scheffold ${ }^{1}$ and Bruno Weber ${ }^{3}$ \\ 1 Department of Physics, University of Fribourg, 1700 Fribourg, Switzerland \\ 2 IROC Science AG, 8005 Zurich, Switzerland \\ 3 Institute of Pharmacology and Toxicology, University of Zurich, 8091 Zurich, Switzerland
}

\begin{abstract}
We present an improved Laser speckle imaging approach to investigate the cerebral blood flow response following function stimulation of a single vibrissa. By synchronising speckle analysis with the cardiac cycle we are able to obtain robust averaging of the correlation signals while at the same time removing the contributions due to the pulsation of blood flow and associated tissue adaptation. With our inter-pulse correlation analysis we can follow second-scale dynamics of the cortical vascular system in response to functional brain activation. We find evidence for two temporally separated processes in the blood flow pattern following stimulation we tentatively attribute to vasodilation and vasoconstriction phases, respectively.
\end{abstract}

\section{INTRODUCTION}

Laser speckle imaging (LSI) is a robust and convenient method to visualise and monitor blood flow in biological tissues $^{1}$. An expanded laser beam is directed onto a section of tissue and the light reflected is detected by the camera's imaging optics. The dynamics of the scattering tissue or particles within a tissue induces a motion of speckle pattern, which blurs the detected image if the camera integration time $\mathrm{T}$ is similar or larger compared to the correlation time of intensity fluctuations. A speckle contrast analysis of the detected speckle pattern provides access to the parameters of the speckle dynamics and of the scatters. By splitting the image into areas and analysing the speckle contrasts within each area one can derive maps of the dynamics. The dynamics can be followed in time with the temporal resolution being set by the camera frame rate.

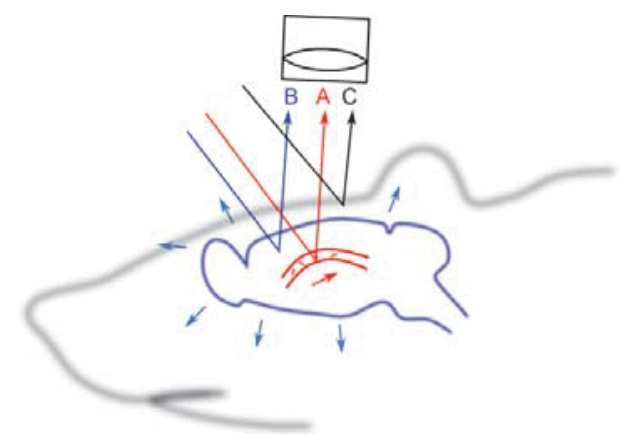

Figure 1 Speckle field back reflected from the brain tissue is formed by the light scattered by the moving blood cells in the micovasculature (A), by the brain tissue matrix (B) and by the static skull (C). Microcirculation is continuous and the speckle field (A) is in continuous fast motion. Speckles (B) are modulated by the cardiac cycle (expansion during early systole, followed by constriction) and vasoactivity caused by activation. Speckles (C) are virtually static.

General LSI uses random fluctuations of speckle fields caused by the motion of light scattered by red blood cells (RBCs). A camera is used to detect the scattered speckle fields with an exposure time $T$ larger than the correlation time $\tau_{c}$ of intensity fluctuations caused by motion $\left(T>\tau_{c}\right)$. The detected speckle field is blurred in the area of interest: more dynamics (i.e. faster or in a larger part of the probed volume) results in greater blurring.

* pavel.zakharov@irocscience.com 
One approach for LSI is based on the quantification of speckle blurring by calculating the contrast of time-integrated intensity distribution $I_{T}(t)$. The time-dependent contrast $K(t)$ is defined as standard deviation $\sigma\left[I_{T}(t)\right]$ normalised with mean $\left\langle I_{T}(t)\right\rangle$, where $\langle\cdots\rangle$ denotes averaging over multiple speckles:

$$
K(t)=\frac{\sigma\left[I_{T}(t)\right]}{\left\langle I_{T}(t)\right\rangle}
$$

The contrast is related to the intercept of the auto-correlation function of time-integrated intensity $g_{2}^{T}(\tau, t)$ in the following way:

$$
K^{2}(t)=g_{2}^{T}(0, t)-1=\frac{\left\langle I_{T}^{2}(t)\right\rangle}{\left\langle I_{T}(t)\right\rangle^{2}}-1
$$

Time-resolved auto-correlation function is obtained from time $T$ integrated intensity as follows:

$$
g_{2}^{T}(\tau, t)=\frac{\left\langle I_{T}(t-\tau / 2) I_{T}(t+\tau / 2)\right\rangle}{\left\langle I_{T}(t)\right\rangle^{2}}
$$

where the estimation of correlation is obtained is at time point $t$ and where $\tau$ is the lag time. The averaging is performed over multiple speckles, which can be resolved in space (as in the classical LSI experiment ${ }^{2}$ ), in time or both.

With an increase in dynamics, blurring increases and the contrast of the speckles decreases, therefore dynamics and contrast are in opposite directions.

Under typical experimental conditions, light is scattered by different structures and tissues: red blood cells (RBC) in the circulation, brain tissue matrix and skull (see Figure 1). All these components contribute to speckle dynamics at different time scales in the detected signal ${ }^{3}$. The flow of RBCs in the blood cause relatively fast speckle fluctuations on the scale of milliseconds and less. Such fluctuations are reduced in a typical LSI experiment due to the intensity of integration during image acquisition and are quantified with speckle contrast analysis as a blurring of the speckle pattern. The amount of blurriness is a characteristic of speckle dynamics and correspondingly of the dynamics of the subject which, in the case of brain imaging, is RBC flow.

Intensity correlation analysis, as applied in a dynamic light scattering framework ${ }^{4}$, contains information from different timescales and thus dynamics on the different temporal scales may be accessed. It has been shown that non-zero integration time $T$ of the intensity trace introduces a triangular averaging of the correlations function ${ }^{5}$. This averaging has a limited effect on the obtained correlation values when ratio $\tau / T$ is above a certain threshold. Accuracy above $10^{-3}$ is achieved with $\tau / T>8$ according to Magatti and Ferri ${ }^{5,6}$. This is the basis of Schatzel's ${ }^{5}$ multi-tau correlation technique which has been used in correlator design for over 30 years. Thus the correlation function for an image acquisition time of $T=10 \mathrm{~ms}$ is basically unaffected for $\tau>80 \mathrm{~ms}$.

It has previously been shown that LSI imaging data can be enriched with statistics of the static contribution ${ }^{3,7}$, which belongs to the opposite end of the timescale analysed with speckle dynamics. In this study, we approach the intermediate regime of the dynamics observed in the living tissue, which although slower than blood flow, is fast enough to be resolved.

The intermediate timescale region is dominated by the basic dynamics of a living being - the pulsation of blood flow and tissue adaptation to the associated periodic changes of blood pressure. For pulsating living tissue, a periodic modulation of the measured signal is an inherent property of the media under investigation. In such a process, neither temporal nor amplitude characteristics are known in advance and presumed to be stable, which makes reliable tracking of the periodicity of the pulsation challenging. 


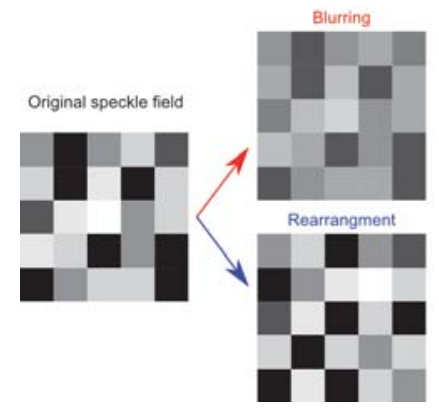

(a)

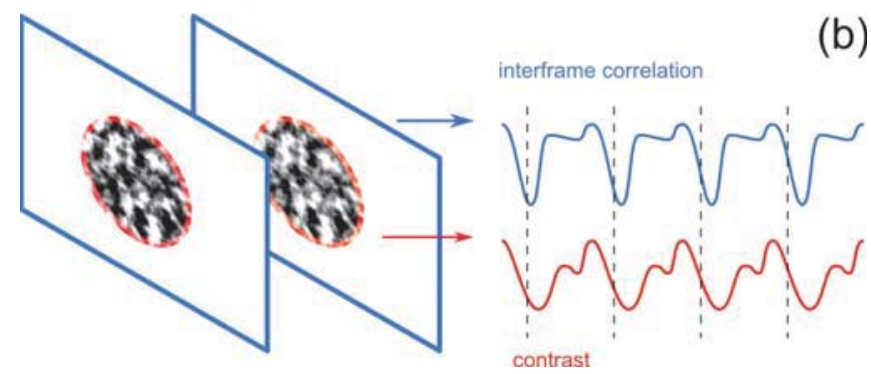

Figure 2 a) Blurring and rearrangement manifestations of speckle dynamics as seen by the camera: Blurring is caused by continuous dynamics on a time scale shorter than the camera exposure, whereas rearrangements are related to dynamics on a longer time scale. b) Contrast characterising sub-integration time intensity variations related to blood flow and inter-frame correlation coefficients, can be used to explore slower dynamics.

However, if successfully implemented, laser speckle analysis synchronised with the cardiac cycle can be used to quantify changes in the speckle pattern from one cardiac cycle to the next and be used to investigate intermediate dynamics of the tissue with significantly improved accuracy.

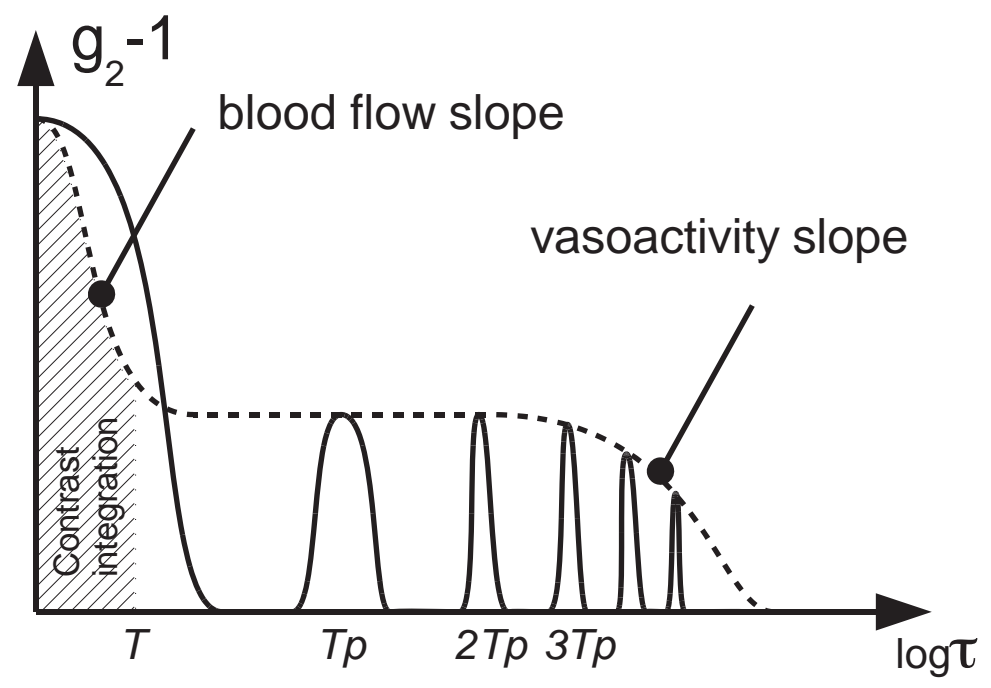

Figure 3 Illustration of the double-slope correlation function modulated with the periodic process. The envelope function (dashed line) obtained without periodic contribution produces a first slope in a short time due to blood flow and the second slope is produced over a longer time is due to vasoconstriction/vasodilation processes. Pulsation modifies the correlation function and allows us to access lag times at multiple pulse periods $T_{P}$. For this illustration we assume a constant pulse period.

As a model for the application of the technique described here, we used the adaptation of tissue matrix to the modulation of blood flow during functional activation of the brain of an anesthetized rat. With heart rate in the range of 250-450 bpm, we expected a minimal inter-pulse time of $133 \mathrm{~ms}$, which is greater than the low accuracy threshold of the multitau averaging $80 \mathrm{~ms}$ introduced above (for a given integration time $\mathrm{T}=10 \mathrm{~ms}$ ).

Three main dynamic processes modify speckle patterns of laser light reflected by brain tissue: scattering from RBC in motion is the fastest process, thereafter the evolution of the speckle field governed by motion of the tissue matrix. The latter process is dominated by the cardiac cycle as the tissue matrix has to adapt to the periodic increase and decrease in blood pressure. We expect the tissue matrix to return to a state characteristic of the specific phase after a complete cardiac cycle. This periodicity can be detected by the peak of the correlation function at the lag time equal to the heart cycle time. Finally, we speculate that vasodilation followed by vasoconstriction during neural activation can be observed as a slow change in the tissue matrix reflected in a speckle pattern evolution from one cardiac pulse to another. 
The dynamics of the brain tissue matrix of an immobilized animal can be observed during expansion and contraction of the early systole with the arrival of a pressure wave. In this case, dynamics is slower and not washed out by the time integration of a camera exposed for $\mathrm{T}=10 \mathrm{~ms}$. Thus, the evolution of the speckle field between images can be observed. Previously, we proposed the dynamic laser speckle imaging (dLSI) method, which allows the separation of dynamic and static contributions to the speckle signal ${ }^{3}$. In the scope of dLSI, slow dynamics can be quantified by the correlation of speckle images separated in time. One challenge in studying slow dynamics of biological tissue, is the instability introduced by the cardiac cycle.

In this paper we present a novel approach to the study of the slow evolution of scattering tissue by cross-correlating speckle patterns from the same phase of the cardiac cycle. To do this, we first solve the problem of reliable detection in the systolic phase of the cardiac cycle. Furthermore, we analyse the similarity of the speckle patterns in consecutive heart beats which allows us to study the slow components of a speckle pattern and thus to investigate the dynamics of the tissue matrix. For a first application, we test whether spontaneously hypertensive rats (SHR) and age-matched WistarKyoto rats can be differentiated with respect to vasodilation and vasoconstriction phase dynamics.

\section{METHODS}

All experimental procedures were approved by the local veterinary authorities in Zurich and conformed to the guidelines of the Swiss Animal Protection Law, Veterinary Office, Canton Zurich (Act of Animal Protection 16 December 2005 and Animal Protection Ordinance 23 April 2008). Surgical procedures were performed under isoflurane anesthesia (2.53.5\%). Animals were tracheotomized and artificially ventilated. The skull above the left barrel cortex was carefully thinned. The animal's temperature was kept constant at $37^{\circ} \mathrm{C}$ with a heating blanket and blood gases were maintained within physiological ranges by adjusting the ventilation when necessary.

Following a baseline of 2 seconds, a single vibrissa contralateral to the thinned skull was deflected for 4 seconds at a frequency of $4 \mathrm{~Hz}$.

Collimated $785 \mathrm{~nm}$ laser light was shone onto the imaged cortical area. Images of the diffusely reflected speckle field were acquired using a 12 bit Pixelfly VGA camera CCD camera (PCO Imaging, Kelheim, Germany) through a stereomicroscope (Leica MZ16 FA, Leica Microsystems, Switzerland) focused $0.5 \mathrm{~mm}$ below the cortical surface. Images with an original resolution of 640 x 480 pixels were acquired with a frame rate of $50 \mathrm{~Hz}$ (inter-frame time $\Delta t=20 \mathrm{~ms}$ ) and an exposure time $\mathrm{T}$ of 10 milliseconds. The f-stop of the imaging optics was adjusted to optimize speckle contrast and signal to noise.

\section{ANALYSIS}

We use the field correlation function $G(\tau, t)$ for a generalised speckle analysis:

$$
G(\tau, t)=\sqrt{g_{2}^{T}(\tau, t)-1}=\sqrt{\frac{\left\langle I_{T}(t-\tau / 2) I_{T}(t+\tau / 2)\right\rangle}{\left\langle I_{T}(t-\tau / 2)\right\rangle\left\langle I_{T}(t+\tau / 2)\right\rangle}-1}
$$

where $\tau$ is lag time and averaging is performed globally over the region of interest defined on the image frame. For $\tau=0$ field correlation function reduces to the contrast: $G(0, t)=K(t)$. The first accessible non-zero lag time equals the inter-frame time $\Delta t$. 


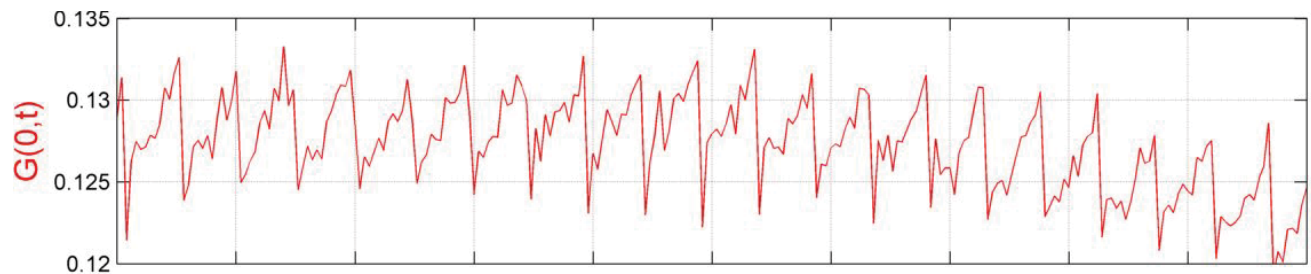

(a)

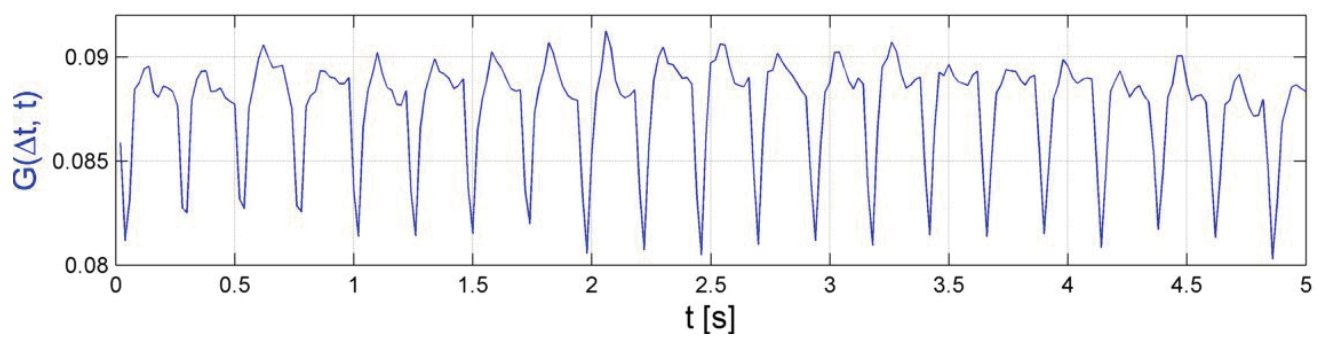

(b)

Figure 4 Pulsation-modulated speckle contrast signal (a) and correlation signal $G(\Delta t, t)$ (b). The contrast signal shows a characteristic drop during systole, indicating increased blood flow, followed by a gradual return to the baseline.

We found $G(\Delta t, t)$ to be the most reliable indicator of pulse phases. Typically $G(\Delta t, t)$ is stable and drops periodically for 20-60 milliseconds due to the macroscopic dynamics of the brain surface caused by the arriving pressure wave from the heart (see Figure 4). With tissue matrix expansion to adapt to the increased blood flow during the early systole, speckle field evolves and correlation signal $G(t)$ decays. This moment is detected in the correlation signal when $G(\Delta t, t)$ falls below a predefined threshold. To eliminate effect of drifts we performed high-pass filtering of the original signal with a Butterworth filter (cut-off frequency of $2.5 \mathrm{~Hz}$ ). The signal is interpolated to achieve a time resolution of $2 \mathrm{~ms}$, we use this trigger to lock the phase to the cardiac cycle. Additionally, the time-dependent period of cardiac cycle, $T_{P}(t)$, is extracted from the time difference between cycles.

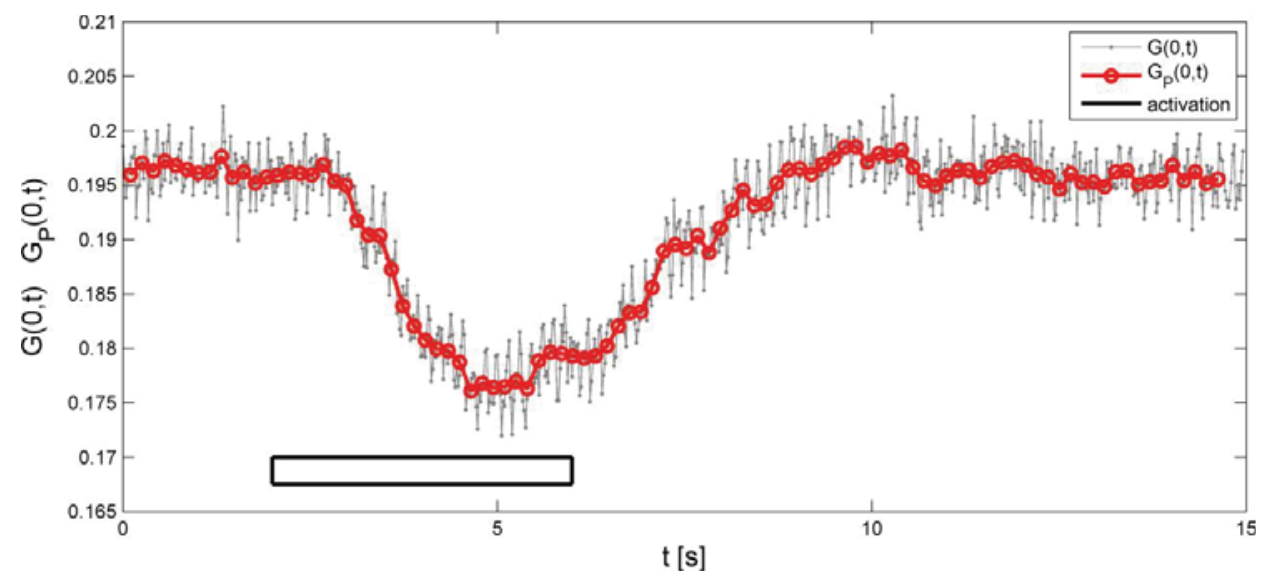

Figure 5 Original contrast time trace $G(0, t)$ and pulse-averaged trace $G_{P}(\tau, t)$. The rectangle indicates activation period.

Using the timing derived in the previous step, we performed speckle analysis of the microcirculation synchronised with the cardiac cycle. Averaging the $G(\tau, t)$ within a heart cycle allows the reduction of characteristic noise of LSI from acquisition at random moments of cardiac activity. We further refer to the cycle averaged signal as $G_{P}(\tau, t)$. Figure 5 is an example of the original contrast signal $G(0, t)$ and the pulse-averaged signal $G_{P}(0, t)$. There is a pronounced decrease of contrast variations with intra-pulse averaging.

For the next level of synchronized analysis we use the period $T_{P}(t)$ to estimate pulse-to-pulse correlation signals $G\left(T_{P}(t), t\right), G\left(2 T_{P}(t), t\right)$ and $G\left(3 T_{P}(t), t\right)$ and to average signals within a single cycle. This provides a time-dependent parameter of the similarity of a speckle field from one cardiac cycle to its subsequent first, second and third neighbours. 
We expect that in the absence of inter-pulse dynamics, the tissue resumes its original state from one pulse to the next and therefore the speckle field remains stable. However, when vasoactive processes initiate changes in the tissue, the speckle field evolves which is manifested in changes of the inter-pulse correlation signals.

\section{RESULTS}

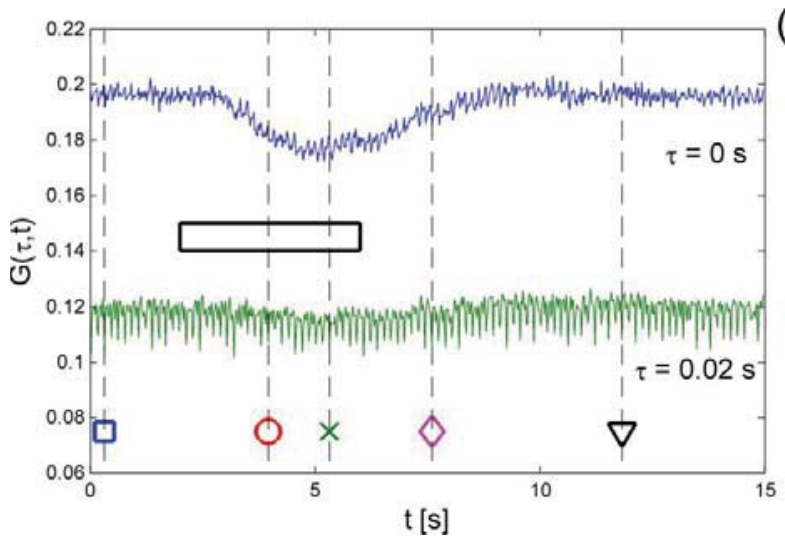

(a)

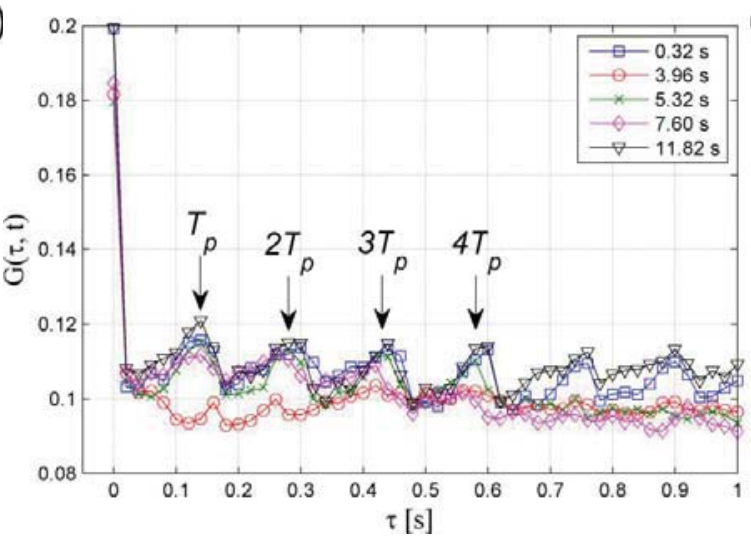

(b)

Figure 6 Example of correlation coefficients changes during functional stimulation (a). Intercept $G(\tau=0, t)$ equals contrast $K(t)$ and $\tau=0.02 \mathrm{~s}$ is the first non-zero lag time. Stimulation time 2-6 seconds is illustrated by a rectangle. Subplot (b) shows full correlation functions obtained at the same phase of cardiac cycle at time points marked on plot (a) at the corresponding symbol. Peaks from the first 4 pulse periods $T_{P}$ are marked with arrows.

Illustration of the signals is presented in Figure 6. We observe a decrease in contrast (intercept of correlation function) during functional activation phase caused by increased blood flow due to brain metabolic demand (Figure 5). Increased blood perfusion leads to a higher blurring of the speckle pattern reflected in the lower contrast. This effect is typically observed in blood flow monitoring with LSI.

For the first non-zero lag time of the correlation function, characterising similarity of the consecutive speckle image $\tau=\Delta t=0.02 \mathrm{~s}$, decay was not pronounced supporting our assumption that the effect of blood flow dynamics is limited to camera integration time $T$. However, a pronounced periodic pattern on the correlation curve was observed, indicating a rapid speckle rearrangement during early systole.

Periodic maxima indicated the reappearance of the speckle pattern after complete cardiac cycles as the largest correlation value occurred at intercept (contrast) (Figure 6b). Surprisingly, not only was the first peak clearly observed on the majority of the selected correlation functions but also on higher order peaks with a comparable amplitude, indicating speckle pattern similarity over an extended lag time range. The amplitude of high order peaks was in addition affected by heart rate variability (HRV), as a large variability of $T_{P}$ in time would smooth out the peaks decreasing the amplitude. In our experiments we detected low HRV caused by anaesthesia.

The minimal baseline reached by correlation functions was well above zero, which is a manifestation of the presence of the speckles originating from static structures, such as the thinned skull.

The largest amplitudes of the first $T_{p}$ peak were observed in the resting phases at the beginning and end of the experiment. The minimal peak amplitude was reached in the middle of the stimulation phase $(t=3.96 \mathrm{~s})$ during vasodilatory evolution of the tissue matrix, with the expansion of the vasculature. A low amplitude was also observed after stimulation ( $t=7.60 \mathrm{~s}$ ) when vasoconstrictory processes were activated and lead to the rearrangement of tissue namely constriction of the vasculature. Finally, at the peak of the haemodynamic response at the end of the stimulation phase $(t=5.32 \mathrm{~s})$, the amplitude of the first peak was similar to the peak during resting phases. This occurred in the interval when vasodilation was complete but vasoconstriction has not yet begun, thus no major long term changes took place. 


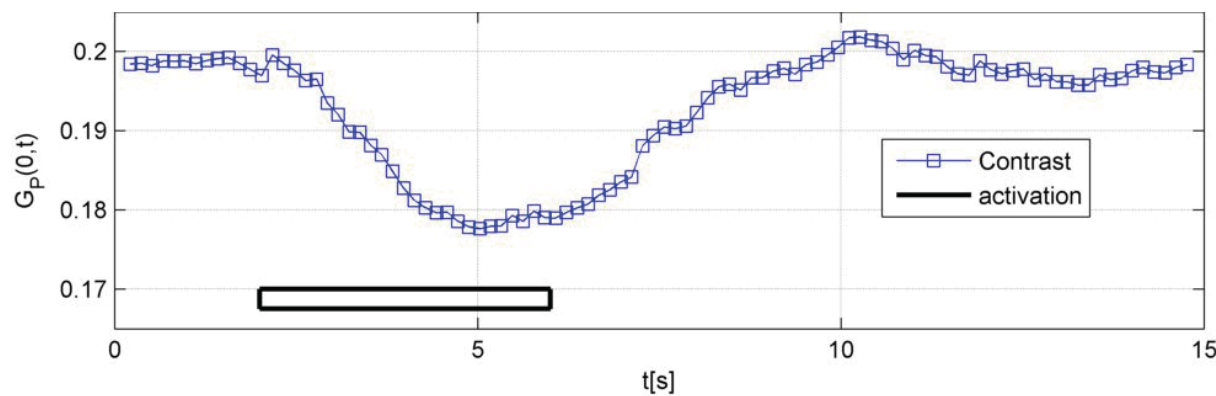

(a)

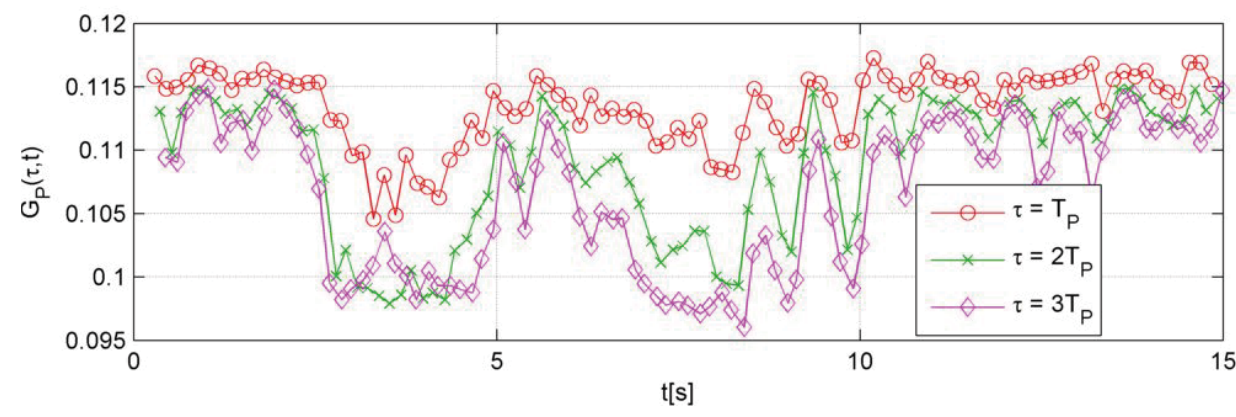

(b)

Figure 7 Pulse-averaged peak amplitudes of the correlation function (a) contrast trace - intercept. The black rectangle indicates activation time. (b) inter-pulse correlation signals: circles - single period lag time, crosses - double period lag time and diamonds - triple period lag time. Each point of the graph represents the average over a single cardiac cycle.

We reviewed the correlation functions measured at a specific phase of the cardiac cycle. As the correlation function changed in relation to the phase of the cycle, we wanted to obtain the average characterising the complete cycle. To achieve this, we used the timing derived from pulse detection and then analysed pulse averaged correlation values.

Figure 7 shows the time traces of correlation coefficients during the activation experiment as illustrated in Figure 6. Pulsation variability was reduced in the curves, with the smallest variability seen in the contrast. At the same time, in the high-order correlation traces we observed new periodicity, which was not observed in the contrast. In this example, the periodical variations of correlation coefficients of $0.7-0.9$ seconds can be attributed to respiration. Respiration can cause in either a limited head motion, variation in blood volume or respiratory sinus arrhythmia, which might not be visible in the pulsation peaks but may be observed in the smoothing of high-order correlation peaks.

The striking difference in the dynamics of inter-pulse correlations was that we observed two increases in activity measured with a corresponding drop in amplitude - during activation and immediately following activation, which we attributed to vasodilation and vasoconstriction phases, respectively. In the current example, the first peak amplitude of the vasodilation phase had a shorter time span and larger amplitude, whereas the vasoconstriction peak was longer and less intensive. 

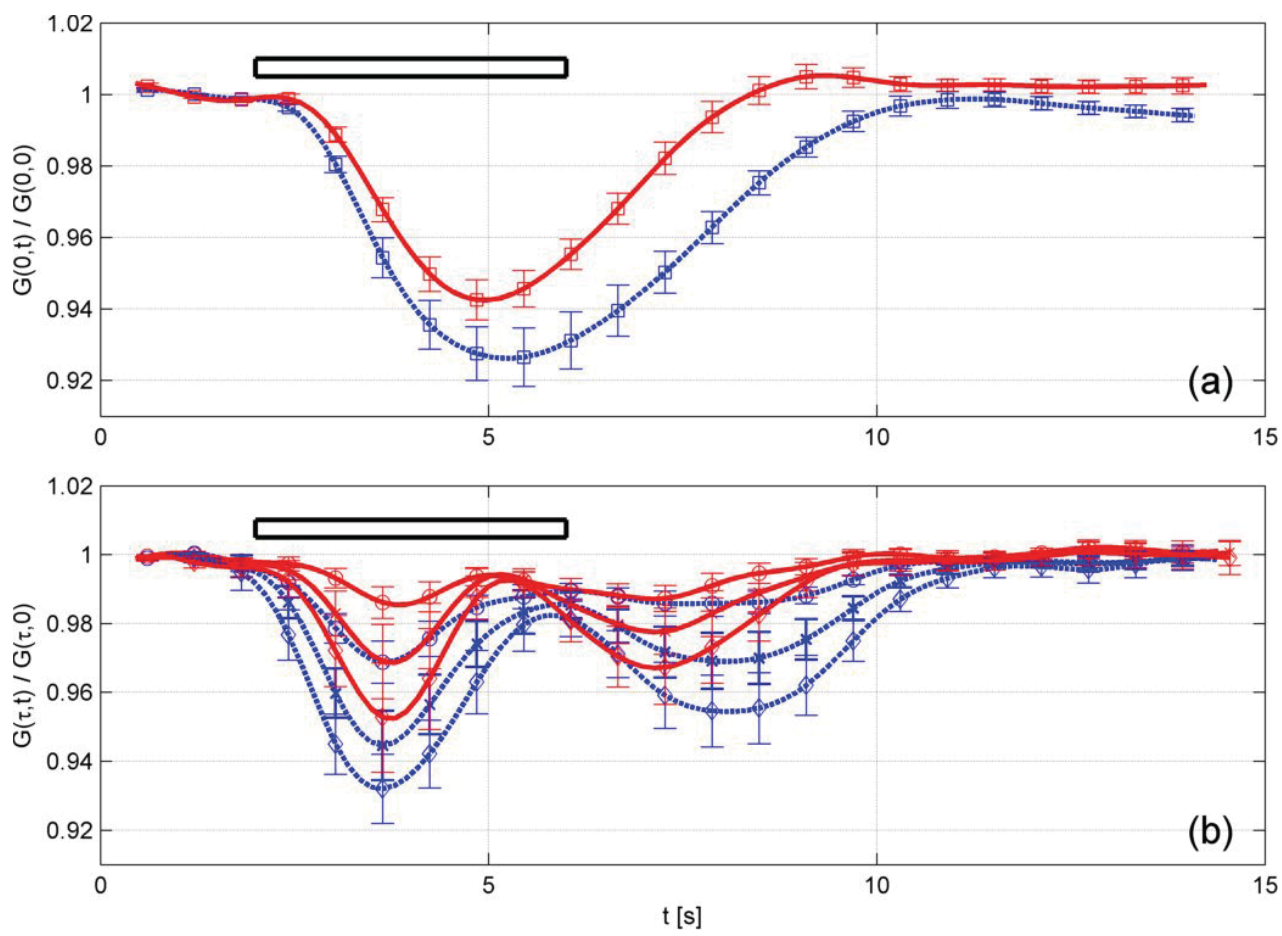

Figure 8 Speckle dynamics averaged over the animals of the SHR group (solid lines) and of the control group (dashed), during functional activation Signals of blood flow dynamics measured with (a) contrast and (b) of tissue dynamics measured with inter-pulse correlation $\tau=T_{P}$ (circles - smallest response), $\tau=2 T_{P}$ (crosses) and $\tau=3 T_{P}$ (diamonds - largest response) . Black boxes indicate stimulation time.

The observed blood flow changes as shown in Figure 8 are typical for functional activation experiments - showing a drop in contrast following the onset of activation and a single pronounced minimum followed by relaxation ${ }^{10}$. Change in signal of the SHR animals was lower compared with the control group, confirming previously reported findings ${ }^{11}$.

As the vasculature should adapt to allow for increased blood flow, we observed the dynamics of the tissue preceding the increase in flow. Thus the correlation signal showed a drop in correlation at around 3 seconds, with maximum flow observed at approximately 5 seconds. We observed a greater response for higher order correlation peaks than expected, as the larger temporal separation between the speckle fields made the signal more sensitive to changes in flow. However, the temporal resolution was remarkably lower for higher order correlations.

The difference between the groups for the vasodilation signal amplitude from the correlation function was greater than for flow response however the timing of vasodilation was very similar for SHR and control groups, as can be seen from the correlation minima.

Furthermore, the amplitudes of the vasoconstriction phase, were different for the groups but the most striking differentiator was the timing of the apogee. The minimum correlation amplitude for the SHR animals was reached at approximately 7 seconds, whereas for the control group it was reached at approximately 8 seconds.

\section{SUMMARY AND CONCLUSIONS}

We present a new approach to the analysis of laser speckle imaging data synchronised with the cardiac cycle. It broadens contrast analysis to a more general framework of correlation analysis derived from well-established techniques of dynamic light scattering and correlation spectroscopy. In this study we develop a technique to use the cardiac cycle inherent periodicity of the living organism to differentiate between the contribution of flow, pulse-modulated tissue motion and static contribution. We were able to track the adaptation of brain tissue in response to functional activation and link the flow response with vasodilation and vasoconstriction activities. It was also possible to differentiate between animals from the hypertensive and control groups. 
In the current study, we did not exploit the spatial resolution of the imaging system. Instead we spatially averaged the detected speckle field to obtain time traces of interest. It is however easily possible to expand the scope of this approach and to perform imaging to investigate heterogeneous dynamics of the tissue matrix ${ }^{9}$.

In conclusion, we propose that pulse-synchronised correlation analysis is a promising new method, combining the simplicity of laser speckle imaging hardware with the power of dynamic light scattering methods. Pulse-synchronised correlation analysis has the potential to provide new insights into dynamic processes occurring within biological tissues.

\section{REFERENCES}

[1] Boas, D. A.., Dunn, A. K., “Laser speckle contrast imaging in biomedical optics.,” J. Biomed. Opt. 15(1), 011109 (2010).

[2] Briers, J. D.., Webster, S., "Laser speckle contrast analysis (LASCA): a nonscanning, full-field technique for monitoring capillary blood flow.,” J. Biomed. Opt. 1(2), 174-179 (1996).

[3] Zakharov, P., Völker, A. C., Wyss, M. T., Haiss, F., Calcinaghi, N., Zunzunegui, C., Buck, A., Scheffold, F.., Weber, B., “Dynamic laser speckle imaging of cerebral blood flow.,” Opt. Express 17(16), 13904-13917 (2009).

[4] Berne, B. J.., Pecora, R., Dynamic Light Scattering. With Applications to Chemistry, Biology, and Physics, Dover Publications, Inc., New York (2000).

[5] Schätzel, K., “Noise on photon correlation data. I. Autocorrelation functions.,” Quantum Opt. J. Eur. Opt. Soc. Part B 2(4), 287-305 (1990).

[6] Magatti, D.., Ferri, F., “Fast multi-tau real-time software correlator for dynamic light scattering.,” Appl. Opt. 40(24), 4011-4021 (2001).

[7] Zakharov, P., Völker, A., Buck, A., Weber, B.., Scheffold, F., “Quantitative modeling of laser speckle imaging.,” Opt. Lett. 31(23), 3465-3467 (2006).

[8] Zakharov, P., Cardinaux, F.., Scheffold, F., "Multispeckle diffusing-wave spectroscopy with a single-mode detection scheme.,” Phys. Rev. E 73(1) (2006).

[9] Zakharov, P.., Scheffold, F., "Monitoring Spatially Heterogeneous Dynamics in a Drying Colloidal Thin Film.,” Soft Mater. 8(2), 102-113 (2010).

[10] Weber, B., Burger, C., Wyss, M. T., von Schulthess, G. K., Scheffold, F.., Buck, A., “Optical imaging of the spatiotemporal dynamics of cerebral blood flow and oxidative metabolism in the rat barrel cortex.,” Eur J Neurosci 20(10), 2664-2670 (2004).

[11] Calcinaghi, N., Wyss, M. T., Jolivet, R., Singh, A., Keller, A. L., Winnik, S., Fritschy, J.-M. M., Buck, A., Matter, C. M., et al., "Multimodal imaging in rats reveals impaired neurovascular coupling in sustained hypertension.,” Stroke 44(7), 1957-1964 (2013). 